\title{
Vorwort des Bergmännischen Verbandes Österreichs
}

Online publiziert am 29. Oktober 2015

Die Montanuniversität Leoben feiert ihr 175-jähriges Bestehen. Der Blick auf diese Zeitspanne dokumentiert eine beispiellose Entwicklung von den Anfängen um 1840 bis zur Gegenwart und verspricht eine überaus erfolgreiche Fortführung dieses Weges in der Zukunft.

In zahlreichen Schriften zu diesem Jubiläum wird dieser Werdegang gewürdigt. Aus dem Blickwinkel des Bergmännischen Verbandes Österreichs, der seit seiner Gründung an der Montanuniversität Leoben seine Heimat hat, soll auch auf die Besonderheit der Berg- und Hüttenmännischen Monatshefte hingewiesen werden.

Der Leser dieses Periodikums findet in jedem Heft zu Beginn den Satz: „BHM - Technisch-wissenschaftliches Organ der Montanuniversität Leoben, der ASMET (Austrian Society for Metallurgy and Materials) und des BVÖ (Bergmännischer Verband Österreichs).”

Seit der Gründung der Steiermärkisch-ständischen Lehranstalt in Vordernberg zeichnet sich unsere Alma Mater durch die Verknüpfung von Forschung und Lehre mit einem eigenen Druckwerk aus. Dem ersten Direktor der Lehranstalt, Peter Ritter von Tunner, ist es zu verdanken, dass 1841 das "Jahrbuch für den innerösterreichischen Berg- und Hüttenmann“ geschaffen wurde. Zu Beginn des ersten Jahrbuchs wird von der feierlichen Eröffnung der Lehranstalt am 4. November 1840 berichtet. Von 1851 bis 1937 kam das Periodikum als "Berg- und Hüttenmännisches Jahrbuch“ heraus. Peter Tunner selbst hat bis zum Jahr 1866 die von ihm herausgegebenen Jahrbücher persönlich geleitet. Seit 1938 erscheinen diese als „Berg- und Hüttenmännische Monatshefte."

Alle diese Bezeichnungen verweisen auf die beiden Urkompetenzen unseres Hauses. In der Zwischenzeit ist die Zahl der in Form von Publikationen in den BHM vertretenen wissenschaftlichen Fachgebiete stark gestiegen. Darin spiegeln sich die bahnbrechenden Entwicklungen an der Montanuniversität wider. Die Breite der Kompetenz in Lehre und Forschung umfasst heute alle Bereiche der rohstoffbasierten Wertschöpfung. Rund 2200 Artikel sind seit 1841 im Rahmen der sogenannten Berghefte erschienen. Der weitaus überwiegende Teil dieser Artikel wurde von Angehörigen und von Absolventen der Montanuniversi- tät verfasst. Damit wurde zum einen der Verpflichtung zur wissenschaftlichen Publikation nachgekommen.

Zum anderen ist die Montanuniversität in ihrer gesamten Geschichte stets geprägt von der intensiven Verzahnung mit der betrieblichen Praxis und den engen Kooperationen mit der Industrie. Dies lassen zahlreiche Artikel in den BHM erkennen, die nicht wissenschaftliche Abhandlungen zum Schwerpunkt haben, sondern die als Erfahrungsberichte aus den Betrieben beschrieben werden können. Nicht zuletzt dadurch schließt sich der Kreis des Wissenstransfers von der Universität in die betriebliche Praxis, von wo die dadurch gewonnenen praktischen Erfahrungen wieder in die Forschung und Entwicklung einfließen.

Der Bergmännische Verband Österreichs ist stolz darauf, Teil der Geschichte der Montanuniversität Leoben zu sein. Bei allen Tagungen des BVÖ, die in Leoben stattfinden, ist die Montanuniversität Veranstaltungspartner. Dabei wird ein wesentlicher Teil der Vorträge von den Wissenschaftlern aus unserem Haus gestellt. Die Zusammenarbeit zum gegenseitigen Nutzen war immer wegweisend und möge es auch in der Zukunft sein.

Wir gratulieren unserer Alma Mater zum 175-jährigen Bestehen und wünschen eine gedeihliche Entwicklung in der Zukunft.

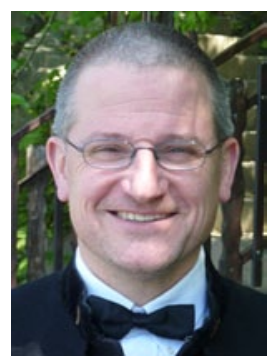

Martin Lang

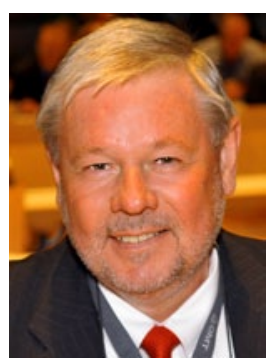

Gerhard Mayer 